Lehren, die dem Prinzip der Privatautonomie zuwiderlaufen, konnte durchaus das eine Renaissance erlebende Naturrecht dienen.

Die Intentionen der Eckhardtschen Studienordnung konnten länger wirken als alle nationalsozialistischen Gesetze je in Geltung waren. Eine Rechtsgeschichte der deutschen Nachkriegszeit ist damit auch immer im Lichte der 1935 eingeleiteten Entwicklung zu betrachten. Man hat den Rechtsunterricht energisch »aus den traditionellen Bahnen « herausgeführt ${ }^{4}{ }^{8}$ und ließ über die weltanschaulich-normative, eben auch politische Wirkungsabsicht keinen Zweifel.

\title{
Helmut Kramer Die Freiheit der richterlichen Meinungsäußerung
}

Wenn Juristen eine strittige Rechtsfrage zu klären haben, greifen sie zuallererst zu einem der Gesetzeskommentare. Wie die dort gesuchte und meist verlässlich gefundene »herrschende Meinung « entstanden ist, interessiert nur wenige.

Einen beachtlichen Beitrag zur Auflösung des Rätsels »h. M.« hat die kürzlich zur Richterin am Bundesgerichtshof ernannte Richterin Gabriele Caliebe geleistet. Schon immer hatten einige unverbesserliche Justizkritiker leise Zweifel an den von den Großmeistern der juristischen Methodenlehre verkündeten These geäußert, die »h. M.« sei das Ergebnis einer allen zugänglichen demokratischen Diskussion um die vernünftigste Gesetzesauslegung, frei von irgendwelchen Vorgaben, sei es durch Rechtsfortbildungskartelle, sei es durch die Zensur in einem zunehmend monopolisierten juristischen Verlagswesen, unabhängig auch von der »Schere im Kopf « aufstiegsbewusster Kolleginnen und Kollegen.

Einen aufschlussreichen Einblick in die Entstehungs- und Stabilisierungsbedingungen der h. M. hat nun Gabriele Caliebe ermöglicht. Gabriele Caliebe gehörte zu den entschiedensten Verteidigern des Rechtsberatungsgesetzes vom I 3. I 2. I 935 . In ihrem gemeinsam mit Günter Rennen (Jahrgang 1926) herausgegebenen Kommentar (3. Aufl. 200I) erklärte sie alle »in der Nachkriegszeit unternommenen Versuche, das Gesetzeswerk als typisches Produkt des Nationalsozialismus darzustellen «, (für) gescheitert. Verfassungsrechtliche Bedenken wollte sie nicht einmal gegenüber dem Verbot der altruistischen Rechtsberatung gelten lassen. Im Bereich der Rechtsberatung sei mit Recht jedwede Betätigung, sei es »aus Nächstenliebe oder sozialem Engagement « verboten. Dazu und zum Beleg, dass bei Verdacht der Wiederholungsabsicht auch eine Einzelhandlung genügen könne, berief sie sich, allerdings ohne das Entscheidungsdatum (1938!) zu erwähnen, auf ein Urteil des Reichsgerichts, wonach die tatbestandsmäßig geforderte Geschäftsmäßigkeit »ihrem Wesen nach eine Frage der inneren Einstellung « sei. Dass das Operieren mit Wesensbegriffen und Gesinnungsmerkmalen aus dem Argumentationsarsenal der Nationalsozialisten, insbesondere eines Carl Schmitt, stammt, geriet ihr nicht in den Blick.

Inzwischen wissen wir es: Was sie da in Druck gegeben hat, entsprach gar nicht der Überzeugung der Autorin. Auf einem Hearing der SPD-Bundestagsfraktion am 5. 6. 2002 mit massiver Kritik konfrontiert, glaubte sie zu ihrer vermeintlichen Ehrenrettung ihre unkritische Darstellung wie folgt entschuldigen zu können:

luste, Kontinuitäten und Diskontinuitäten in den Wirtschafts-, Rechts- und Sozialwissenschaften zwischen den $20 e r$ und soer Jahren, Stuttgart i 998 , S. I $33 \mathrm{ff}$.

48 Wieacker (Fn. 7). 
»Richterin am OLG Köln Gabriele Caliebe erörtert die rasante Entwicklung des RBerG, welche durchaus als negativ betrachtet werden kann. Sie selbst halte das Gesetz eindeutig nicht für zeitgemäß und vor allem im Hinblick auf die Ausführungsverordnungen nicht mehr für praktikabel. Diese Kritik könne sie jedoch nicht immer in der Kommentierung anbringen, was auch an den Vorgaben des Beck Verlages liege. Kritik habe sie im Bereich der unentgeltlichen Rechtsberatung. Sie selbst verstoße bei den Ratschlägen in der Nachbarschaft ständig gegen das RBerG. Ihr bliebe dann nur die kleine Hintertür des Begriffs der »Geschäftsmäßigkeit«, der durch Wiederholungsabsicht beschrieben werde. Also sage sie sich jedes Mal, sie würde dies »nie wieder tun «, so dass die Wiederholungsabsicht fehle. Jede weitere Nachbarschaftshilfe sei dann ein neuer Fall. Das sei natürlich nicht witzig, da es sich um eine Ordnungswidrigkeit handele, obwohl Anzeigen bei der Staatsanwaltschaft gewöhnlich im Sande verlaufen. (...) Für sie stehe außer Frage, dass die altruistische oder karitative Rechtsberatung freizugeben sei. ${ }^{\mathrm{I}}$

Es bleibt sich gleich, ob der Beck Verlag tatsächlich, wie manche schon immer geargwöhnt hatten, die von der Autorin behauptete Zensur ausgeübt oder ob nur vorauseilender Gehorsam im Spiel gewesen ist oder die »Schere im Kopf «, die hier die wahre von der veröffentlichten Meinung getrennt hat, jene Neigung also, den allgemeinen Meinungstrend mit der eigenen Meinung zu verwechseln. Anspruch auf Autorität kann ein solcher Kommentar jedenfalls nicht erheben. Zugleich bezeichnet das unfreiwillige Geständnis der Verfasserin das Elend einer von der Interessenlobby gesteuerten Kommentarliteratur als Produktionsstätte der »h. M.«.

Bei den beiden anderen Kommentatoren des RBerG sind wir auf Selbstbezichtigungen nicht angewiesen: Die Herausgeber des das RBerG einbeziehenden Kommentars zur BRAO (2. Aufl. 2003, Verlag C. H. Beck), die Professoren Dr. Martin Henssler und Hanns Prütting, sind die Direktoren des Instituts für Anwaltsrecht der Universität Köln. Dies Institut wird von der Hans-Soldan-Stiftung finanziert, hinter der der Deutsche Anwaltsverein (DAV) steht. Der Verfasser des soeben erschienenen Kommentars Chemnitz/Johnigk (RBerG, I I. Aufl. 2003), der gleichfalls an dem alten Meinungsstand festhält und die neueren kritischen Stimmen unterdrückt, ist Geschäftsführer der Bundesrechtsanwaltskammer - ein weiteres Beispiel für die interessengebundene Art, in der die sog. »h. M.« lenkt, wenn nicht manipuliert wird. Unter Aufgebot komplizierter Umschreibungsformeln vermeiden alle Kommentatoren geflissentlich auch jeden klaren Hinweis auf die problematische Tatsache, dass die Erlaubnisfähigkeit für die unentgeltliche Rechtsberatung im Jahre I 980 völlig abgeschafft worden ist. Die Geschichte des RBerG einschließlich der Verlogenheit der angeblichen Gesetzeszwecke ist eine Geschichte der Verdunkelung (Kleine-Cosack spricht von einer »wissenschaftlichen Nacht « um das RBerG), der Unaufrichtigkeit und des Konformismus. Um so bedauerlicher ist die unkritische Hinnahme des Gesetzes und seiner erweiternden Auslegung in den Kommentaren durch die Gerichte. Nur so konnte es dazu kommen, dass ungezählte altruistische Helfer wegen »unerlaubter Rechtsberatung « verurteilt worden sind.

Glaubt man den Apologeten des RBerG, ist es schon den Nationalsozialisten bei dem Verbot der altruistischen Rechtsberatung in wohlmeinender Absicht um nichts anderes gegangen als um den »Schutz der Rechtssuchenden vor unsachgemäßer Rechtsberatung «. Tatsächlich trägt das Gesetz unverkennbar den Stempel seiner Herkunft: Als die Nationalsozialisten daran gingen, Anwaltschaft und Justiz von jenen zu trennen, die sie als »jüdisch«, » marxistisch « oder als sonstige »Volksschädlinge « bezeichneten, hatten sie vor allem die im Auge, die den vom Unrecht bedrängten Bürgern hätten rechtlichen Beistand leisten können. Das »Rechtsberatungsmissbrauchsgesetz « - so der ursprüngliche Name - wurde erlassen, um den aus »rassischen« oder politischen Gründen aus dem Amt gejagten Rechtsanwälten die 
letzte Ausweichmöglichkeit zu nehmen. Auch sollten sie daran gehindert werden, "Staatsfeinden « mit Rechtsrat zur Seite zu stehen - ein Gesetzeszweck, den die Machthaber diskret mit der »Sicherstellung eines reibungslosen Ablaufs von Verwaltung und Rechtspflege « umschrieben. ${ }^{2}$

Zurück zur Autorin Gabriele Caliebe. Inzwischen ist sie, bislang Richterin am OLG Köln, zur Richterin am Bundesgerichtshof ernannt worden, unter vorläufiger Abordnung an das Bundesministerium der Justiz. Dort soll sie nämlich die mit der Reform des RBerG befasste Mannschaft verstärken. Hatte sie sich für diese Karriere vor allem durch ihre redlichen Bemühungen um das RBerG empfohlen?

Doch sollte man der neuen Bundesrichterin nicht vorwerfen, als einzige gegen den Komment verstoßen zu haben. Gibt es nicht allzu viele Rechtswissenschaftler und Richterkollegen und -kolleginnen, die sich gegenüber Erwartungshaltungen nachgiebig zeigen, sei es aus Karrierewünschen, sei es um des lieben Friedens willen, auch wenn sie nicht immer so »laut denken« wie Gabriele Caliebe? 